\title{
Data preservation and risk management in Management Information Systems
}

DOI: $10.46932 / \mathrm{sfjdv} 3 \mathrm{n} 1-112$

Received in: Jan 30st, 2021

Accepted in: Feb 1th, 2022

\author{
Sampath Chandrasena \\ PhD Reading, MSc. in Information Security \\ Institution: Ch Charan Singh University, Meerut, (UP), India \\ Address: No 349/2, Kahatagastenna, Ruwanwella, (71300). Sri Lanka \\ E-mail: schandrasena@gmail.com
}

\begin{abstract}
Companies have indeed been able to cut down, widen their market coverage, as well as shape relatively close working relationships while working to improve client relationships thanks to technological processes. However, using the Internet has introduced new risks and concerns. This research highlighted the problems that CIOs and IT managers face in the field of the organization. It covers data security in its newest manifestation, effective management issues, safety disciplinary measures and methodologies, and more. Risks from cyberspace are also a concern. It also develops a threat classification model and also regulatory systems. It also employs a few explanations to develop a system for calculating the impact of security risks in a statistical sense. It entails determining the likelihood of successful information attacks. The paper also evaluates the physical and perceived expenses of the known vulnerabilities, and some recommended control laws and simulation methods for measuring the injuries done by such infringements. This study as well begins to develop a five-stage risk mitigation system for evaluating incidents involving information management: 1- Appraisal of the resource's and application's worth; 2Remotely exploitable and risk assessment; 3-Computation of threats-related losses and the advantages of preventive actions; 4- Choosing a surveillance system and; 5- A simulating different strategies. The research findings must aid judgment in deciding the best controls to reduce safety issue detriment. Finally, a few recommendations for further research have indeed been put in place to improve protection. Massive quantities of assets and analysis in organizations, as well as assessing the chance of successful potential attacks on these investments. This study created an overarching risk management system for security analysts, allowing them to better focus their efforts to include the most appropriate control measures. There are five parts to this scheme: Commodity and application value explanation, Vulnerability review, Loss arithmetic following complaints and advantages of control legislation, Done through a process collection, and Implementation different alternative appraisal. Fenceline routers, numerous different intrusion prevention, security systems, firewalls, and scheme records are identified as countermeasures for maybe the first danger; password protection and malware barcode readers have been identified as an effective control law again for Remotely exploitable and risk assessment; and eventually, an essential tool for managing, firewalls, and assertive methodologies are identified as effective control measures for denial - of - service.
\end{abstract}

Keywords: data preservation, risk management, mis, information security.

\section{INTRODUCTION}

The increasing amount of Global Internet commercial transactions is being threatened by legitimate complaints about the safety of systems with a large number of potential systems that affect. 
Despite the potential benefits of trying to conduct transactions over the Internet, some companies have indeed been slow to adopt new tech. The possibility of losing investments and confidentiality as a result of potential security breaches in such systems may be the strongest motivation for households and companies to avert attempting to define and participate in e-commerce. For example, a solitary wellpublicized security vulnerability can erode confidence in a corporation and have far-reaching ramifications in the e-commerce sector, to not mention harm to the future of the company. The present state of security setup in organizations.

The core of security concerns, the significance of tracking data security senior executives' practices to ensure that their measures are fully retained and revised, as well as how to enforce effective internal controls to rebut people.

\section{OVERVIEW OF RESEARCH}

Valid concerns about the safety of a system with a large range of possible systems that affect are threatening the massive increase of World Wide Web commercial activity. Given the advantages of doing business online, some businesses have indeed been slow to adopt this new tech. The risk of losing investments and confidentiality due to potential security vulnerabilities in such processes is the most convincing reason because both companies and organizations avoid trying to establish and partaking in etransactions (e-commerce). A solitary, well-publicized security flaw, for instance, can corrode trust in a company and cause massive consequences in the e-commerce sector, not to mention harm to the company's reputation.

\subsection{RESEARCH PROBLEM}

This research establishes some tradeoff analysis methods for considering the prices of security protocols to infraction rate/reliability/safety metrics. It also suggests a method for determining how adequate control measures are.

\section{OBJECTIVE}

This study as well begins to develop a five-stage risk mitigation system for evaluating incidents involving information management:

1- Appraisal of the resource's and application's worth

2- Remotely exploitable and risk assessment

3- Computation of threats-related losses and the advantages of preventive actions

4- Choosing a surveillance system and 
5- A simulating different strategy the research findings must aid judgment

\section{ORGANIZATIONAL PRACTICE AND THREATS}

Information security is defined as "the security of data against Security can be described as "the protection of data against data leakage, transfer, reconfiguration, or destabilization, as to if unintentionally or done on purpose," according to Federal Benchmark 1037C. (1997). Data security is a continually evolving field. Security is recognized as an important aspect of any information system which requires great consideration, starting with the system design and continuing thru the entire production process. Failing to acknowledge security standards correctly can consequence not only in vulnerabilities, and also civil proceedings and outright denial of the good or service by customers. Companies are constantly trying to figure out how to understand the risks to their data resources of how to get the assets they have to combat them.

The more we investigate the current state of business, the more we discover the practice of data protection The essence of security issues as well as how to allocate resources in data security are then discussed by management consultants.

\subsection{BUSINESSES' PROTECTION PROCEDURES}

The State of the Art The vast majority of companies use they have implemented their information security programs in a piecemeal and poorly integrated manner. In an idealistic situation, the security management function is governed by a management consultant who indicates the general impact of the information security management system to an executive management team advisory board. This paper concentrates on the exclusions and forthcoming adjustments to the preliminary story when that program is in position.

There will be no prescriptive information or directions for experts combined with the network security plan's general rules, objectives, and preconceptions for customers. Framework guidelines ("subscribers should authenticate") will be perceived as policy requirements including such "we then would manage customer information mystery." a unique password that is changed at least once every 90 days").

Most companies have an "awareness program " in place to educate employees about the firm's security protocols. The data security software, and also how to recognize and notify an issue, are covered in an introductory module for new hires. A second module covering updates is provided to staff and construction companies. When those who start the job or the surroundings dramatically change, they should notify the information security management system. Every employed person must participate in a 
one-hour average yearly upgrade symposium. Data security product lines and instruments are sourced via a direct Request for Planning (RFP) navigator by a constant evaluation process that assesses the disparity between policy requirements and platform capabilities. Such tools are only used after they have undergone extensive testing.

A public statement guiding their usage objectives is developed by the chief 8 security officer, who has an obligation for the architectural style. A computer hackers rescue squad (generally a simulated team comprised of just a few key IT experts) usually responds whenever a data security incident is presumed to evaluate logs, configuration files, causing considerable obstructions. Regular authorization issues have been addressed by an automatic vehicle convenience and availability (new, changed, or deleted). Password reset cases are made by the personality function. Eventually, as part of a comprehensive inspection, the whole data security system is checked and validated in light of technological changes, the e-commerce platform, riskiness, working population demographic trends, and corporate structure. Only a fraction of the aspects of a successful corporation information security program is generally accomplished by business owners. The program was launched. Massive, public corporations understand this.

The principal information security department, which is in charge of architecture, creates a memorandum that overpowers one's utilization aims. Whenever a data protection event is suspected, a computer hackers rescue squad (generally a simulated team comprised of just a few key IT experts) works fast to assess logs, internet connectivity, and seeking to establish. Regular connect cases are made by an automated provisioning system (new, changed, or deleted). Password reset cases are made by self-service functionality. Eventually, as part of a comprehensive review, the whole data security program has been checked and validated in light of changes in technology, the marketplace, risk profile, workforce demographics, and corporate structure. Only a portion of the components of an effective enterprise-wide information security strategy is typically implemented by business owners. Training program. Huge, public corporations understand this.

3 components can occur as a consequence of a data security breach:

1) Brand and reputational harm,

2) Legal and regulatory risk, and

3) Economic ramifications

In associated with information security protocols, the next layer of companies is much less suited and much more informed, reliant on the skills from one or multiple information technology experts who are using their skills and experience to implement how much they believe to be a suitable collection of control legislation. A lot of small businesses are unlikely to take significant measures to protect or retain 
their IT facilities. Data security expenditure, excluding disaster recovery, typically accounts for less than $3 \%$ of the IT spending plan, according to Gartner Group facts and figures.

Financial support for implemented components of the information security management system is only warranted in the event of a cause's surface, such as virus protection in the event of computer viruses or routers in the event of problems with web implementation. A training and awareness software could include an amusing story about just a recent problem that's been expected to deal with it after the reality, instead of a cooperation strategy invented first before the issue arose. As a consequence, no learnings in improving corporate culture were managed to learn. The vast majority of companies could be completely unaware of their security vulnerabilities, allowing security flaws to go undiscovered for extended periods.

Experienced professionals are taught how and where to minimize the effects of a cyber-security breach and also how to framework efficient and unambiguous performance management in universities. Even though, in the daily grind of company or organization, these lessons are usually ignored. It requires assets to complete computer printout processes, overviews supposedly finished work, enforce extra security measures, reboot sufficient default username and password, remove unutilized scheme ids, or pertain spots to troubles that have not yet occurred. Firms frequently concentrate on relatively brief strategic problems while overlooking the corporate strategy consequences of an action. Expenses can be reduced, unforeseen brownouts are lowered, and fulfillment is managed to improve whenever a natural monopoly in a provided geographical location or sector tries to implement an integrated and comprehensive information systems security.

Correspondingly, companies have recognized that the good processes facilitate improved regular audits and coaching, but few companies document even their core procedures thoroughly. Most leaders know that wasted time, effort, resources, and products are costly; however, few are willing to go to the trouble of having a comprehensive review of their methods for determining and reducing wastage, data duplication, and service interruptions. In the 1960s, the American industry-endorsed performance as a separate and distinct action plan. Senior executives at major manufacturing firms were given the title of "Directors of Quality" and given the task of lowering defective products. These unfortunate individuals were completely ineffective even though those who did lack the organizational clout to modify the workflow. Processes in relation include design, development, manufacturing, and field preservation.

Information security is a consideration in the specification, design, development, deployment, and maintenance of information technology. Throughout the lifecycle of the system, a comprehensive information security program must be deeply integrated. The architecture, design, development, deployment, operations, and maintenance of an information technology environment determine its information security characteristics. After an information technology environment has been deployed, 
information security cannot be added. Information security is a property of an environment rather than a product.

Correspondingly, companies recognize that well-documented mechanisms facilitate improved regular audits and coaching, but few companies document even their core procedures thoroughly. Most leaders know that wasted time, effort, resources, and products are costly; however, few are willing to go to the trouble of having a comprehensive review of their methods for determining and reducing wastage, data duplication, and service interruptions. In the 1960s, the American industry-endorsed performance as a separate and distinct action plan. Senior executives at major manufacturing firms were given the title of "Directors of Quality" and given the task of lowering defective products. These unfortunate individuals were completely ineffective even though those who did lack the organizational clout to modify the workflow. Processes in relation include design, development, manufacturing, and field preservation.

Effectiveness is not even an output factor in the production process, according to the study results of all these failed metrics. We later found that satisfaction is an essential component of product innovation. To put it in another way, the value of a manufactured object is determined by the manufacturing process.

Performance, unlike acrylic, cannot be decided to add after an object has been completed. Similarly, we're beginning to realize that InfoSec isn't a distinct field from computer technology. The configuration, layout, advancement, rollout, and maintenance of information systems all take safety into account. Comprehensive security software should be highly intertwined all through the program's entire life cycle. Data security properties are determined by the architectural history, layout, advancement, implementation, operational processes, and upkeep of a world of information technology. Sensitive information could be decided to add after an information systems climate has indeed been implemented. Instead of being a result, InfoSec is a characteristic of surroundings.

\section{THE MANAGEMENT INFORMATION SYSTEM (MIS):}

Is an interconnected man-machine system that gives data to help supervisors organize and control their organizations? Change is a method used to describe a group of people Management can be characterized as a feature, a procedure, a career, and a social group. It consists of a variety of tasks or activities that responsibility is shared for. The particular element of the operations is ascertained by these organizational processes as organizing, organizing, trying to direct, governance, and attempting to control.

1. Starting to plan: This is the process to determine ahead of time what actions to take and when and where to take them. Its goals in the most efficient manner possible, as well as for forecasting potential issues and risks as well as another process improvement 
2. MIS is a scheme that assists governance in creating, having to carry out, and attempting to control choices," says Jerome Kanter. Planning is the process of formally clustering different activities to enable the achievement of organizational goals.

3. The goals of the farm It is important to apply commitments, employment, and hierarchies to employees.

4. Controlling: This entails progress towards the achievement of strategies and trying to correct any variances which may take place.

5. Guiding: This is the method of placing proposals, structures, and group efforts into action inside the intended way. It is required for the successful execution of a project by having the needed management inspiration and interaction.

The leadership can be divided into three levels based on their hierarchical structure:

- Key Operational or top management

- Strategic or middle management

- Organizational or junior management

Top management establishes the policies, plans, goals, and expenditure framework wherein the institution's different departments will function.

Middle management is in charge of putting top management's laws and long-term plans into action.

Junior Management is in charge of carrying out whatever policies and decisions are made by management positions to create products and services that meet income, financial gains, as well as other objectives.

MIS is an interactive user device system providing information to support operational control, management control, and strategic decision functional areas, as per Davis and Olson. Resources such as hardware, software, manpower, procedures, and suppliers are used by information systems.

\section{MIS ORGANIZATION AND MANAGEMENT TRIANGLE FRAMEWORK}

In 1965, Robert Anthony proposed that management planning and control be classified into three parts. These are the following:

1. Make the strategic plan.

2. Operational management.

3. Management oversight. 
The planning process begins to develop the approach for determining the organizational mission and new breed of applications to those goals, as well as developing policies to regulate asset sourcing, use, and temperament. Managerial control: It is required by managers of different teams to ensure that everything is to assess performance, determine corrective action, develop new decision rules, and allocate resources Operational control is the process of carrying out operational activities to make the best use of resources. It employs pre-determined procedures and decision-making rules.

\subsection{DATA PRESERVATION}

The Blue Ribbon Task Force on Sustainable Digital Preservation and Access (2010) states that digital information is a valuable resource in our knowledge economy, with applications in research and education, science and the humanities, creative and cultural activities, and public policy. Massive amounts of data are being generated by new high-throughput instruments, telescopes, satellites, accelerators, supercomputers, sensor networks, and simulations (Thanos, 2011).

Decision-makers use these statistics to improve citizens' quality of life. Furthermore, researchers are analyzing these data using sophisticated technologies to answer previously unanswerable questions (Helbing \& Balietti, 2011). Massive datasets, unprecedented levels of openness among researchers, and new connections between researchers, policymakers, and the general public have all been facilitated by digital technologies. And the general public (The National Academy of Sciences, 2009).

However, digital data is inherently fragile and is frequently lost.

1. Users may be unable to comprehend or apply the information.

2. Information may become inaccessible due to a lack of long-term hardware, software, or computer environment support;

3. Evidence may be lost due to the origin and authenticity of the data.Loss of the ability to identify where data is stored,

4. The current data custodian, whether an organization or a project, may go out of business at some point in the future

5. The people we entrust with our digital assets may fail us.

To counter these threats, digital preservation aims to keep digital information accessible, independently understandable, and usable by a designated community for the long term, with evidence proving its authenticity (Giaretti, 2011). 


\subsection{LITERATURE REVIEW}

The number of information studies highlights the benefits of information gathering, extracting, compendium, connecting directly, and allocation, with little attention paid to the risks associated with data collection, harvesting, compilation, access, and distribution (Clarke, 2016). As a consequence, the purpose of this research on risk assessment for data leakage or lost opportunity prevention in MIS is to fill a gap in the literature. The writings on financial planning in MIS are analyzed in this chapter, with a focus on data breach and loss prevention. In the second part, the author establishes the study's theoretical foundation. MIS Risk Assessment Ideas and Procedures because today's systems are so complicated, they are vulnerable to security breaches. Portfolio management systems are needed by organizations that want to minimize exposure to benefit from the clear benefits of some good data systems many business owners failed to adequately start enforcing and adherence risk mitigation relating to information systems given the complexity of financial planning. (Yan and Sauter, 2013).

Major corporations are becoming highly dependent on information systems, even though any risk management blunder could result in harm ranging from a minor "annoyance" to a "catastrophe" (Loch, Carr, and Warkentin, 1992, p. 173). When it comes to risk planning, there are six major blunders that companies make. The very first mistake is those who are inclined to predict risks based on the historical data (Stulz, 2009; Taleb, Sprtznagel, and Goldstein, 2009). This is a problem because businesses now operate in a dynamic, rapidly changing market. As a consequence, any danger that existed in the past may or may not be relevant in a future risk assessment. For instance, Bilge, Han, and Dell'Amico (2017, for example) propose a risk management plan that incorporates previous experience. User security awareness, usage patterns, and the overall vulnerability of the data and its storage location are all factors to consider.

The second blunder is that organizations frequently assess risks using "narrow measures." For example, focusing solely on financial risks will not provide companies with a comprehensive picture of the data risks they may face. "Erroneous managerial decisions" are frequently made when organizations take a narrow approach to risk assessment (Myskova and Doupalova, 2015, p. 330). Third, businesses frequently overlook or "overlook" common risks. Risk management in information systems, according to Sherer and Alter (2004), necessitates the identification, definition, conceptualization, and organization of all potential risks following their potential for harm.

As a result, disregarding a danger will adversely affect Risk Firm's current effectiveness in terms of data safeguard. Fourth, organizations struggle to maintain information service risk when "concealed dangers" are overlooked or ignored. Eventually, managers may struggle to connect or disregard recognized risks to the appropriate personnel. If business owners intend to carry out one's risk management plan my duties are on a "real-time basis," those who danger horrendously mismanaged risks 
(Stulz, 2009; Taleb, et al., 2009). Because of the fast rate of knowledge advancing technology as well as the reality that its information appears to apply to and affects each area of business, real danger governance in MIS is essential. As just a consequence, portfolio management programs will be both obligatory and adequate.

Because of the fast rate of knowledge advancing technology as well as the reality that information appears to apply to and affects each area of business, real-time risk leadership in MIS is critical. As a consequence, threat assessment programs will be both reasonable and necessary in helping to understand, evaluate, and inhibit "dangers, security flaws, and security mechanisms" with such a sensible amount of assurance (Nikolic and RuzicDimitrijevic, 2009, p. 595). As a consequence, anything below real-time monitoring and management would be inadequate, and exact risk assessments and classification will be hard to come by.

Data breaches in the recent past have demonstrated the need for accurate data preservation risk management in MIS. There's no denying that data is important leaks are a serious threat to today's businesses. When sensitive data is leaked, businesses can suffer immediate and long-term financial and reputational consequences can be seen too.

A data breach costs a business at least US\$4 Risk Management Data Prevention 14 million on average. Juniper Research recently predicted that global data breach costs will reach US\$2.1 trillion billion by the end of 2018. (Cheng, Liu, and Yao, 2017). As information harvesting becomes more digitalized, these losses are unavoidable. Yahoo revealed in 2016 that 500 million accounts had been compromised in 2014. (Cheng, et al., 2017). With the increased use of digital technologies, these types of data breaches and risks have become more serious. Without a doubt, the digitalized era is opening up new and exciting business and social opportunities, but it also poses significant risks that must be addressed (The Royal Society, 2016). As a result, the requirement. It is now more important than ever to have adequate risk management programs. According to a recent study conducted by Centrify and Ponemon (2018), data loss and/or breach hurt consumer loyalty and shareholder value.

As a result, businesses must figure out how to manage risks to protect customer data from actual loss or theft. A company's reputation suffers immediately after a data breach. According to the researchers, Centrify and Ponemon (2018), the company affected loses 5\% of its stock immediately after a data breach is revealed to the public. With the global economy becoming more interconnected and trade between developing and developed countries increasing, risk management for data breach prevention is becoming increasingly important for all consumers and businesses around the world. While businesses in developed countries may implement and use efficient and effective risk management systems, data breaches in other countries will have an impact on their operations and customers. 
Security Threats in Information Systems: Classification We need a good threat classification scheme before we can analyze security threats and develop a scheme or methodology for allocating resources and deploying control measures against them. "Any taxonomy used to price security failures should be deterministic and complete," according to the taxonomy to be used in this study. There should be no unclassified security failures, and no security failure should fall into more than one classification" [10]. In general, categorizing a phenomenon allows for systematic research. An organized classification of e-commerce threats, in particular, can assist managers in developing less vulnerable systems. When reporting incidents to incident management, having a classification system in place would be beneficial.

Examining Existing Taxonomies: A literature search revealed multiple attempts to categorize security risks. The Naval Research Laboratory created a taxonomy that divides security flaws into three categories: genesis (deliberately or inadvertently caused), introduction time (during development, maintenance, or operation), and location (software or hardware). Neumann and Parker [48] devised a ninecategory classification system for computer crimes methods (that are ordered from the physical world to the hardware and software and from unauthorized use to misuse of authority, etc). This category appears to cover the vast majority of known techniques, including possible threats and unauthorized users abusing their privileges. It does, however, have some flaws when it comes to categorizing intrusions into one of two classes, or both. Wiretapping, impersonation, and infringements of text secrecy, to name a few.

As per Pfleger, network infrastructure dangers include message integrity violations, hacking, code integrity, and denial of service. Even though all these categorizations cover the most important computer security threats, this author believes that they either do not cover all of them or do not allow them to be considered separately.

\section{METHODOLOGY}

Threats and Safeguards are divided into categories. Putting a Modeling to Work a connectivity system's dangers are examined from two perspectives: threat agent and permeation method. A risk is evidenced by a malicious attacker who uses a particular permeability method to create a negative correlation. Agents that are harmful to the general environment, sending, and unauthorized users are the three types of malicious attackers. Evaluate the two environmental factors: Environmental aspects need to be considered, even though this is basic decency. Ecological factors and environmental catastrophes have a greater impact on some regions than others. Forest fires, for example, are not geological formations reliant, so although tornadoes and river flooding, for example, can be anticipated more commonly in particular places. There is also a natural setting to consider. 
Figure 1- Skill Level- Insider Knowledge Matriz

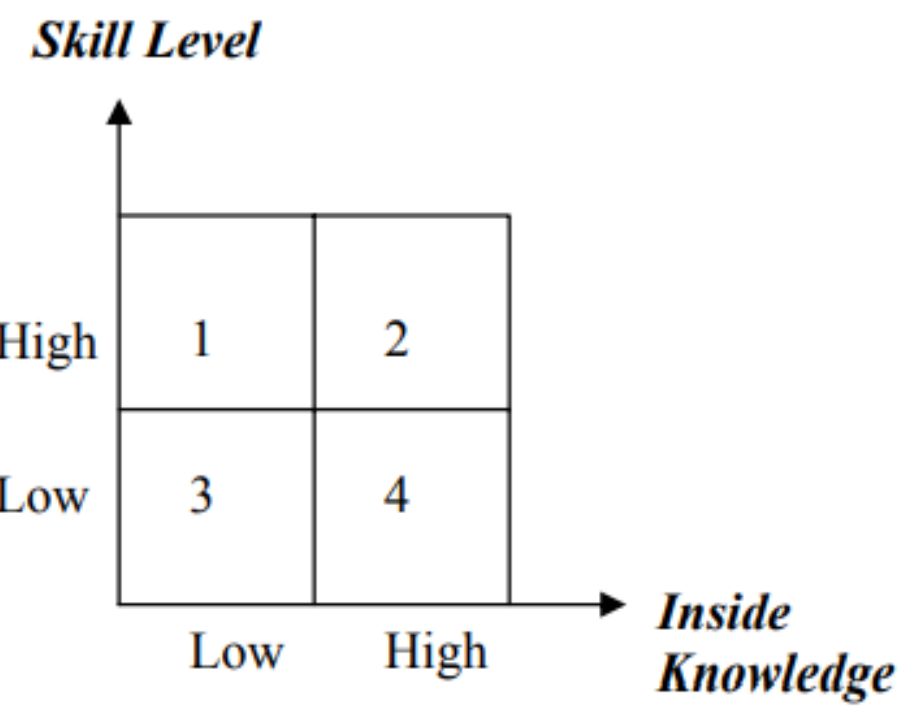

Danger Categorization and Control Policy Dangers to a communication network are studied from two perspectives to use a technology demonstrator: risk advisor and permeation method. A potential hacker creates a risk using a particular permeability method to define an uneasy connection. Injury researchers The three different types of malicious attackers are numerous environments, authorized consumers, and unauthorized access. Examine the following two external factors: Even though it must go on without simply stating, it is important to realize to incorporate environmental factors. External conditions and natural catastrophes have a greater impact on certain regions than others. Flames, for example, are unchanged by geographic location, whereas storms and river flooding, for example, can be anticipated more commonly in particular places. In addition to natural environs, Priority should be given to the risks of design and industrial equipment malfunction, as well as rolling blackouts, in the event of a disaster.

If authorized personnel and employees are involved in providing the abuse of its services one's special rights and officials or make stupid mistakes, jeopardizing the platform's capacity to finish its objective, they may be considered possible risks. Employees with access privileges or in important group roles who do have the capacity or chance to misuse one's connectivity officials, perks, or beliefs must be considered possible threats. Unauthorized users include: Intruders are who are not involved in helping processes or who, on purpose, try to sabotage the scheme or operation's economic output, anymore implicitly or explicitly. Blatant methodologies may include out and out acts of vandalism against equipment and related devices. Covert methodologies are much more nuanced efforts at ruination that can be conducted out by the use of a variety of tools manipulation of software, both systems, and applications, via the internet 
Techniques The following categories apply to tangible, manpower, equipment, applications, and due process methods: Use of physical necessary to acquire access to unauthorized including such building structures, chemical halls, or other public places is known as physical permeation. Staff: Service members who are awarded a few amounts of access and advantage to a scheme, either as customers or technicians, are subjected to permeation approaches and practices. Technicians include analyzing the needs, software developers, insight coordinators, as well as other professionals. They could be recruited by a malicious attacker to penetrate a scheme, procedure, or institution, or they might become aggrieved or energized enough to release theirs possess strike. Equipment: Device threats can be hung using the hesitating using the scheme to try to subvert or vanquish it direct assaults on the hardware, a bug transplanted in a device console, or a strike on the sponsoring utility companies are all instances of trying to exploit the equipment's qualities to thwart the scheme.

Direct assaults on the equipment, a bug transplanted in a device console, or a strike on the sponsoring utility companies are all instances of exploiting the equipment's qualities to thwart the scheme. In this classification, any piece of hardware that is part of the system is regarded as equipment (i.e., the mainframe, peripherals, communications controllers, or modems). The list includes power supplies, conditioning systems, backup generators, as well as other oblique scheme-associated equipment. The software package, program files, and power procedures are all objectives for application permeation methods. Software theft can vary from small differences intended to compromise the system to even more blatant adjustments intended to cause loss of data or even other substantial repercussions.

Protocol: Failure to adhere to existing controls or the dearth of or lack of adequate control system can allow one to take or unauthorized users to pierce the scheme. Procedural infiltration includes ex-staff keeping including using legitimate passcodes, unauthorized service members trying to pick up the outcome, and consumers perusing without even being discovered due to an inability to tirelessly verify audit logs. On a much more granular level, the ISO 7498-2 Standard identifies five cyber security steps to combat such dangers:

The 5 stages in the verification process are
1) Identification,
2) Data secrecy,
3) Access,
4) Integrity of data, and 5) quasi. 
Figure 2- Combination of agentes, techniques, and control measures

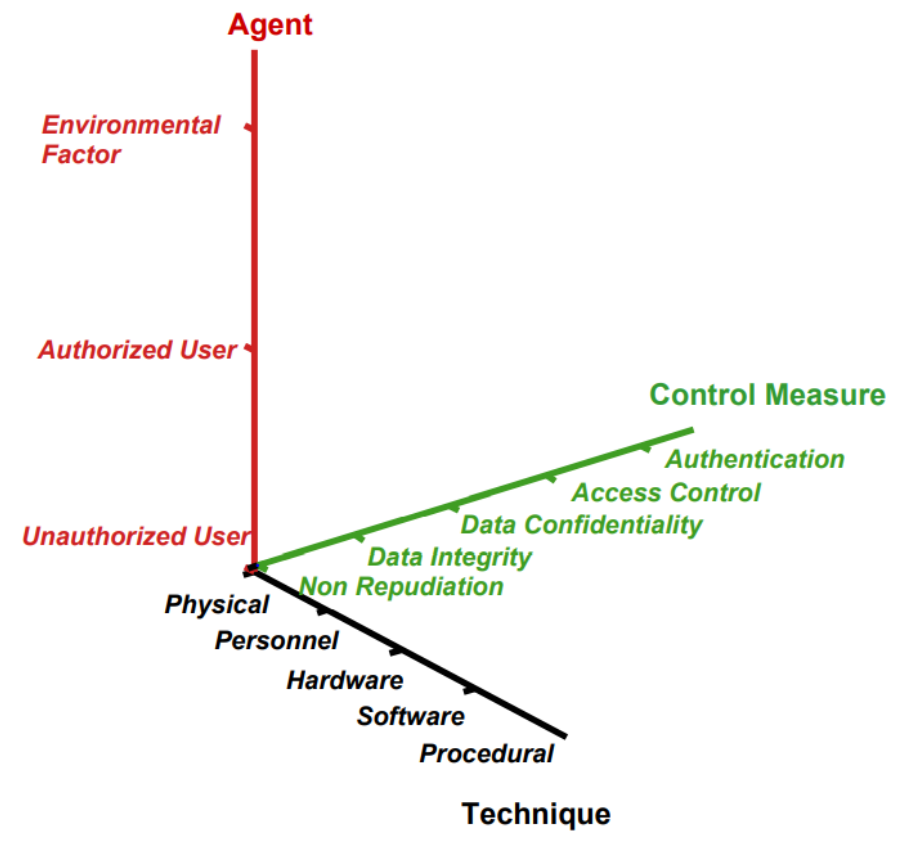

Desktop security professionals generally accept the above categorization, and so this writer supports it as an effective control way of measuring. Verification. The process of associating an identity with a person.

The external device must provide information that will enable the system to verify its identity. This information could be gathered from one or more of the following sources:

1- What the entity knows, such as passwords or sensitive information

2- Possessions of the entity, such as a card

3- The entity's characteristics, such as fingerprints or retinal characteristics

4- The entity's location, such as in front of a particular terminal The authentication process entails obtaining authentication information from an entity, analyzing the data, and determining whether it is linked to that entity. Kerberos and X.509 are two popular authentication protocols in network security. Kerberos is a widely used encryption-based authentication protocol.

An authentication algorithm and an authorization facility are stipulated. The latter allows users to retrieve credentials for public keys, which allows a group of users to trust the public keys' validity. This facility is used in a variety of applications as a core component. Anonymity. The concealment of information or resources is referred to as confidentiality. Data can be obtained in a variety of ways, including wiretapping, planting bugs in output devices, sifting through trash cans, monitoring electromagnetic radiation, bribing key employees, inferring one data point from other values, or simply requesting the data. Information security is a serious concern in data security because data is frequently available in a form that people can read. There are numerous safeguards in place to ensure confidentiality. 
As a result, confidentiality procedures rely heavily on trust and assumptions. The two primary methods for preserving privacy and verifying encrypted messaging are PGP (Pretty Good Privacy) and S/MIME (Secure/Multipurpose Internet Mail Extension). PGP is a widely utilized virtual trademark and encoded communication systems software program that guarantees the privacy of electronic mail sent over the Internet. Consumer authentication tokens are managed using a social security card important management architecture. S/MIME is very similar to PGP, except for the symmetric encryption technique. By mutual authentication with all eligible persons, each customer creates a trusting guy having received the ring. For encoding and decoding, the S/MIME study was designed to assess whether a registration certificate is reliable and valid. Certificates of enrollment are usually described in X.509 format. As a result, S/MIME eradicates a need for exchanging data between the transmission and reception.

Security access measures ensure that privacy is maintained. One authentication scheme for protecting privacy is cryptographic protocols, which makes it difficult to find information to make it unfathomable. In the case of network safety, connectivity power refers to the ability to restrict and limit access to dedicated server applications and systems via communication links. So every organization 59 trying to allow access should first be recognized or accredited, to customize access privileges to the ordinary person. Data consistency. Authenticity is a term that encompasses the reliability of the data collected or assets, and it is typically characterized in terms of avoiding unauthorized or unseemly adjustments. There are 2 kinds of authenticity: data integrity (information content) and integrity of origin (the source of data). Truthfulness methods are divided into two categories: preparedness processes and restoration processes. As well as detection mechanisms that are restored Integrity of data are retained by attempting to prevent unauthorized information changes or data changes that are made unlawfully.

Detection mechanisms do not attempt to prevent data integrity violations; instead, they simply report that the data's integrity has deteriorated. To detect problems, detection mechanisms may check to see whether requisite or anticipated limitations are in place by looking at critical systems, user as well as scheme behavior, or, extra frequently, the data on its own. The processes may document either as the real cause of a truthfulness contravention (a particular component was started changing) or that the file is becoming corrupt. In many ways, continuing to work with truthfulness varies from continuing to work with secrecy. The data seems to be either flawed or not before it did come to secrecy; even so, completed accordingly the information's rightness as well as its truthfulness. The data's origin, what from whom it was acquired, and how it was filtered are all factors to consider.

Non-repudiation precludes a statement from being disputed either by the sender or the receiver. As a consequence, the recipient can prove that a notification was sent from the accusation recipient when it is received. Correspondingly, when delivering a signal, the recipient can show that it did receive by the 
intended user. Such protective measures, and also representatives and methodologies, are depicted in Figure 2. This schematic could be used to categorize dangers to data systems (agents as well as methods), and also security procedures to help counter people. For instance, one of the protective measures being used to combat dangers posed by installing malware customers is the security system. There are 535 threat technique combinations in a combined amount.

Even though some of these combinations, although many of them are relevant, not among them are. For instance, non-repudiation could indeed defend against risks caused by environmental variables or due process methods. This tri representation of potential attackers, methodologies and safety measures to control can aid in the quantifiable evaluation and monitoring of risks. Figure 3 depicts a virus, a denial of service attack, and influencer abusive behavior of internet connectivity as examples of risks and suggested standard precautions inside the prototype.

Figure 3- Virus, denial of servisse attack, and insider abuse of net access in our classification

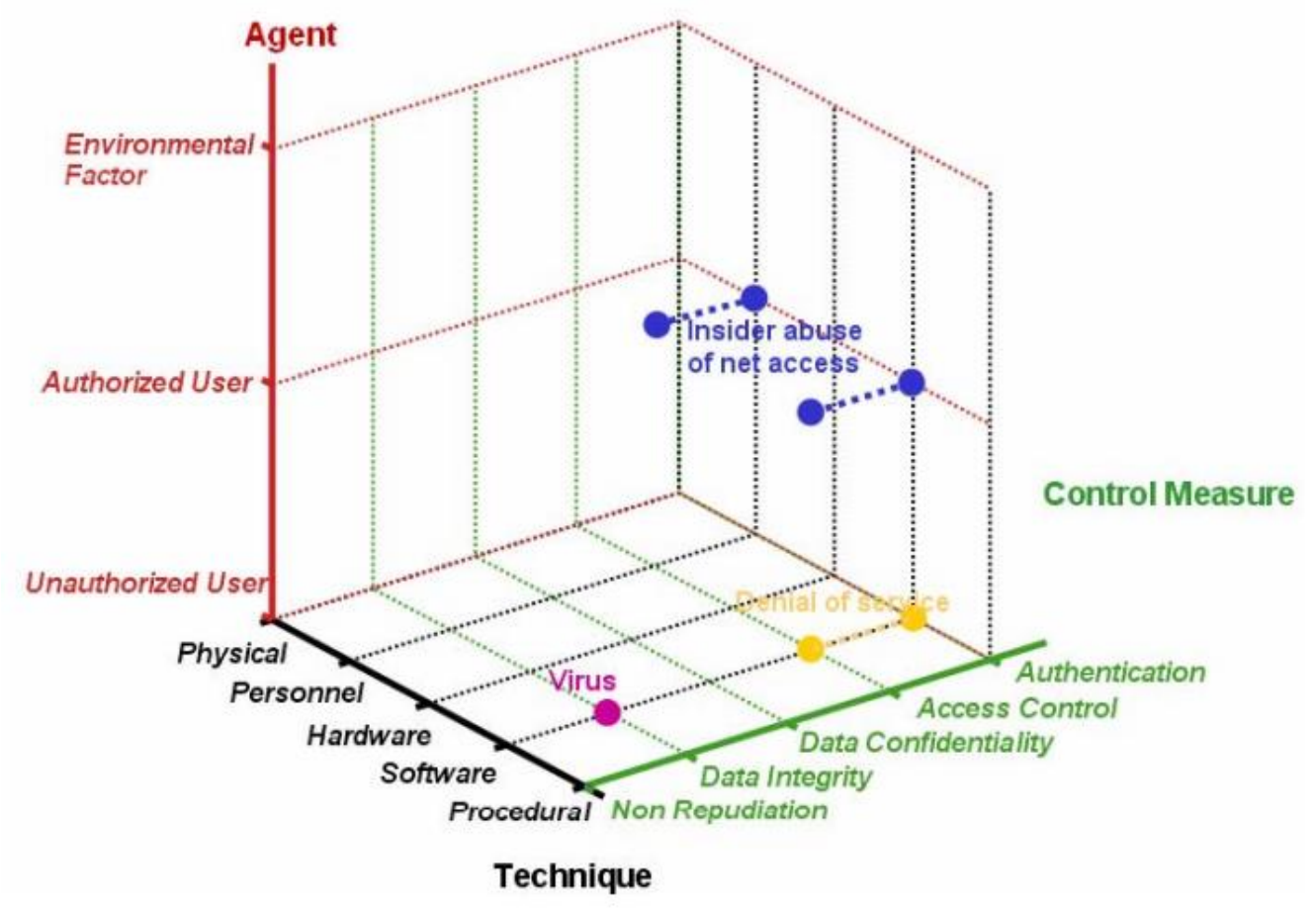

\section{DISCUSSION}

This research examines a few of the vulnerabilities that an e-commerce business is facing, and also management guidance about how to cope with them. The present state of InfoSec practice is investigated in this research. It helps explain how businesses choose where to implement data protection assets as well as how the expenses of previous instances vary by a corporation. As a consequence, "standardizing" a process or continuing to develop a truly unified method for predicting the expenses of security flaw metrics and harm is challenging. Leadership, strategy, architectural style, training and awareness, new 
tech, internal audit, tracking and reporting, and verification are suggested as the last group of components to take into account in an improved mechanism.

Data security initiative A measurable to intangible contrast Now next location of ability to contribute is intangible costs identified from cyber security incidents. As per the results of this study, which were premised on a comprehensive study, individual interviews, and case analysis, the effect of an information technology security event on an organization may be economic in terms of direct damages and expenses, but lengthy unquantifiable harm may demonstrate being more severe. Strong brand, general populace notoriety, intangibles in the global market, and general populace and purchaser self-belief in the precision of business activities are all unquantifiable responsible for damage that can result from the disclaimer of private information.

This research examines a few of the security problems which an e-commerce company may face, and also management advice about how to cope with people. The present state of data security practice is investigated in this study. It tries to explain what businesses choose how to deploy data security assets as well as how the expenses of previous instances vary by the corporation. As a consequence, "standardizing" a process or continuing to develop a truly unified method for predicting the prices of security flaw metrics and harm is challenging. Leadership, strategy, architectural style, training and awareness, new tech, internal audit, tracking and reporting, and verification are suggested as the last group of components to take into account in an efficient plan.

Data security initiative the next area of donation is a comparison of tangible and intangible costs reported from information security incidents. As per the result of this research, which was based on the comprehensive review of the literature, individual interviews, and case analysis, the effect of a cybersecurity event on an organization may be economic in terms of direct damages and expenses, but lengthy unquantifiable harm may prove to be more severe. Strong brand, general populace notoriety, goodwill inside the global market, and general populace and consumer self-belief in the precision of commercial transactions are all unquantifiable harm that can result from the disclosing of private information.

In comparison to hundreds of millions of dollars in managed service threats, this undefinable amount of damage might cost a lot of money. The adjustment of a person's subjective evaluation to evidence-based data in the context of the field of cyber security is the industry research 3rd way of contributing. Order to reduce the impact of a breach of security official statement on an organization, data gathering for cyber security incidents is challenging. This research proposed person's subjective evaluation as a potential substitute. The NIST guidance for leaderboard dangers, the NSA's 18 areas of data security assessment, and eventually, security vulnerabilities best practices are all suggested for helping an organization predict the likelihood of occurrences. This research investigates a few problems. 
Established risk categorizations have flaws and control provisions for information management, and it looks at dangers from two aspects:

Clear and present danger entities are divided into two categories: hazard agencies and risk techniques. Cyber attackers might include the professionals, tangible, machinery, operating systems, and operational; risk (infiltration) methods could include manpower, physiological, machinery, operating systems, and operational. Verification, password protection, the confidentiality of information, integrity of information, and non-repudiation assistance are proffered as countermeasures against such threats. This research established a holistic risk management framework for security professionals, enabling them to effectively redistribute wealth and identify the most appropriate standard precautions. There are five major components to this scheme:

1-Analysis of the source of energy and application value

2- Vulnerability assessment

3- Calculation of failure as a result of risks and the advantages of countermeasures

4- Selecting reasonable regulations, and

5- Assessment of implementation alternatives

\subsection{RESEARCH QUESTIONS:}

Question 1: What would you believe the much more significant threat(s) to your firm's data system are?

Answer: "One of the most serious problems we face is indeed the disclaimer of consumer data,".We are seeing a lot of consumer private information and relate using certain data that all those clients tend to favor to remain secret due to such facets of our business strategy. The destruction of corporation private information is the 2 nd most major threat."

Question 2: How often within the last twelve months have you just been exposed to the type of threat/incident?

Answer: Case 1: "This hasn't happened within the last twelve months." Definite aspects of our IP rights are frequently placed at client site."

Case 2: "Malware, on median wage, one real attack per 30 days." On median wage, one invasion occurs every 6 months. There haven't been any major DoS attacks yet."

Question 3: What kind of harm did the above risks do? (or is likely to trigger)?

Answer: Case 1: "If such an attack were to happen, the corporate image would suffer significantly, but not irreversible, harm." The extent of the harm would've been determined by the buzz surrounding the direct exposure." 
Case 2- "Virus: ended up causing scheme closures and builds new." Incursions: costs of confirmation, external advising, and re-design."

Question 4: Would it be more probable that unauthorized or authorized users will use operating system methods to end up causing this or these dangers?

Answer: Case 1: "Approved users of the system are unlikely to cause these issues because they're often externally energized and have little motivation to participate in these behaviors." Unapproved assailants are far more dangerous."

"Intruder" is the second case.

Case 3: "It may be both. Furthermore, ethical hacking could be used."

Case 4- "For the time being, we're concentrating on unauthorized users, but authorized users remain a source of concern."

\subsection{SIGNIFICANCE OF THE RESEARCH}

The following are the implications of the study:

- All participants indicated the fraud and disclosing of proprietary information as a major threat.

- Viruses, denial-of-service attacks, malicious insiders, weak password protection, and equipment failure also were noted as potential threats.

- No more than one major attack per month, with one intruding every six months on median income.

- All participants probably expect one large-scale attack in the next 12 to 24 months.

- The expense of such an invasion would be determined first by the attack's media exposure, and then by the costs of downtime, notify, consulting, and re-design.

- The most significant risks to an organization that can be induced by software applications have been recognized as unauthorized users.

- The majority of participants were unable to specify which control they were

\section{CONCLUSION}

For the first risk, perimeter access points, numerous intrusion detection, and prevention systems, password protection, security software, and framework records have been recognized as effective approaches; for the 2nd risk, password protection and malware barcode scanner have been reported to be effective control legislation; and then for denial of service, security systems, intrusion prevention systems, and assertive methodologies have been recognized as effective approaches in this research. 
- As per this investigation, trying to make relevant data failures general populace will indeed create the right environment for the formation of a cost-effective data security industry. At the moment, such circumstances need not emerge.

- Trying to make dollar data from being lost accessible to the public will provide a comparable financial for putting in place efficient screening procedures.

- Providing evaluations to personal top management and insurance adjusters. Worth a total will indeed serve as a motivator to enhancing information security protocols. 


\section{REFERENCES}

1. Alberts, C., Dorofee, A., Managing Information Security Risks, the Octave Approach, SEI Series, Addison Wesley, 2003.

2. Anderson, R., "Why Information Security is Hard- An Economic Perspective", 17th Annual Computer Security Applications Conference, Dec. 2001.

3. Bennett, S.P., Kailey, M.P, "An application of qualitative risk analysis to computer security for the commercial sector", Eighth Annual IEEE Computer Security Applications Conference, Nov.-4 Dec. 1992, pp.64-73.

4. Bishop, M., Computer Security, Art and Science, Addison Wesley, 2003.

5. Blakley, B., "The Measure of Information Security is Dollars", Workshop of Economics and Information Security, May 2002.

6. DeMillo, R., Dobkin D. P., "Recent Progress in Secure Computation", The IEEE Computer Society's Second International Computer Software and Applications Conference, Nov.1978, pp. 209-214.

7. Farahmand, F. and Navathe, S.B., A Security Risk Management Model for Electronic Commerce and Security, in preparation, 2004.

8. Lindqvist, U., and Jonsson, E, How to systematically classify computer security intrusions, IEEE Symposium on Security and Privacy, 1997, pp. 154-163.

9. Pfleeger, C. P., Security in Computing, Prentice Hall, 1997.

10. Power, R., Computer Security Issues \& Trends, 2002 CSI/FBI Computer Crime and Security Survey, Vol. VIII, No. 1, Spring 2002.

11. Schneier, B., "No, We Don't Spend Enough", Workshop of Economics and Information Security, May 2002.

12. Wood, Charles C., et. al. Computer Security; A comprehensive Control Checklist, John Wiley \& Sons, 1987.

13. R.Chandra (2018), Management information system 